\title{
LINEAR VS. NONLINEAR EFFECTS FOR NONLINEAR SCHRÖDINGER EQUATIONS WITH POTENTIAL
}

\author{
RÉMI CARLES
}

\begin{abstract}
We review some recent results on nonlinear Schrödinger equations with potential, with emphasis on the case where the potential is a second order polynomial, for which the interaction between the linear dynamics caused by the potential, and the nonlinear effects, can be described quite precisely. This includes semi-classical régimes, as well as finite time blow-up and scattering issues. We present the tools used for these problems, as well as their limitations, and outline the arguments of the proofs.
\end{abstract}

\section{INTRODUCTION}

This paper is a survey of some recent results on nonlinear Schrödinger equations with potential. A particular attention is paid to the case where the potential is a second order polynomial. In this case, the fundamental solution is known explicitly, through a generalized Mehler's formula; the linear dynamics is well understood. The most important remark is that we can assess the action of some Heisenberg observables (which can be exactly computed) on a class of nonlinearities. With this tool, we can understand the interaction between the linear effects caused by the potential, and the nonlinear effects. In the semi-classical régime $\varepsilon \rightarrow 0$, we emphasize some critical scales measuring this interaction, and describe the critical phenomena. When $\varepsilon=1$, we observe the effect of such potentials on finite time blow-up; the potential may "create" some blow-up (think of the harmonic potential), or delay, even prevent, this phenomenon ("repulsive" harmonic potential).

We give two motivations to study nonlinear Schrödinger equations with potential. The first one arises from physics, for Bose-Einstein condensates. The model involves the equation

$$
i \hbar \partial_{t} u^{\hbar}+\frac{1}{2} \hbar^{2} \Delta u^{\hbar}=V(x) u^{\hbar}+a \hbar^{2}\left|u^{\hbar}\right|^{2 \sigma} u^{\hbar} ; \quad x \in \mathbb{R}^{n}, a \in \mathbb{R}, \sigma \in \mathbb{N},
$$

where the role of the potential $V$ is to confine particles. The cases most currently considered are when $V$ is quadratic (isotropic or anisotropic harmonic potential), when $V$ is lattice periodic, or when $V$ is the sum of two such potentials (see e.g. 45 2 [37, 17]). We shall not discuss the physical relevance of this model, but notice that explicit mathematical formulae are available in the case of the harmonic potential. Our main motivation is more from a mathematical point of view. The presence of a potential alters the propagation of the wave in the linear case. Similarly, nonlinear problems may lead to typical phenomena, such as finite time blow-up. How can these two effects interact? This vague question seems to raise many complex issues. We give some very partial answers, essentially restricted to the case where the potential is a second order polynomial. These examples may be viewed as a first step for a general study, supporting or contradicting the intuition. This may be compared with the approach of N. Burq, P. Gérard and N. Tzvetkov, who analyze

2000 Mathematics Subject Classification. Primary: 35Q55; Secondary: 35A05, 35B30, 35B35.

The author acknowledges support by the European network HYKE, funded by the EC as contract HPRN-CT-2002-00282. 
the role of geometry for nonlinear Schrödinger equations on compact manifolds (see e.g. 4, 5]).

The initial value problem we study is

$$
i \varepsilon \partial_{t} u^{\varepsilon}+\frac{1}{2} \varepsilon^{2} \Delta u^{\varepsilon}=V(x) u^{\varepsilon}+\lambda^{\varepsilon}\left|u^{\varepsilon}\right|^{2 \sigma} u^{\varepsilon} \quad ;\left.\quad u^{\varepsilon}\right|_{t=0}=u_{0}^{\varepsilon},
$$

where $\varepsilon \in] 0,1], x \in \mathbb{R}^{n}, V \in C^{\infty}\left(\mathbb{R}^{n} ; \mathbb{R}\right), \lambda^{\varepsilon} \in \mathbb{R}$, and $\sigma>0$ with $\sigma<\frac{2}{n-2}$ if $n \geq 3$ (the nonlinearity is $H^{1}$ sub-critical). We consider two régimes for the parameter $\varepsilon$ :

- The semi-classical limit $\varepsilon \rightarrow 0$. This régime gives hints to understand highfrequency phenomena and provides us with tools which are extremely useful in the nonlinear case, when $V$ is a second order polynomial.

- The case $\varepsilon=1$. This case is better understood thanks to the semi-classical analysis. We study in particular the global existence issue and two of its companions: finite time blow-up and scattering.

We shall not discuss any regularity issue here, and always assume $u_{0}^{\varepsilon} \in \Sigma$, where

$$
\Sigma:=\left\{f \in \mathcal{S}^{\prime}\left(\mathbb{R}^{n}\right) ;\|f\|_{\Sigma}:=\|f\|_{L^{2}\left(\mathbb{R}^{n}\right)}+\|\nabla f\|_{L^{2}\left(\mathbb{R}^{n}\right)}+\|x f\|_{L^{2}\left(\mathbb{R}^{n}\right)}<+\infty\right\} .
$$

We do not discuss the question of solitons either. We denote

$$
H_{V}^{\varepsilon}=-\frac{1}{2} \varepsilon^{2} \Delta+V(x) ; U_{V}^{\varepsilon}(t)=e^{-i \frac{t}{\varepsilon} H_{V}^{\varepsilon}} \text {, and simply } H_{V} \text { and } U_{V} \text { when } \varepsilon=1 \text {. }
$$

This paper is organized as follows. Section 2 is devoted to general results. First, we recall some classical results in the case $V \equiv 0$ and $\varepsilon=1$, as well as some techniques to prove them. We then discuss which ones can be easily generalized when $V$ is not identically zero, and present some cases where a change of variables makes it possible to relate the case $V \equiv 0$ to the case where $V$ is not trivial.

In Section 3 we recall the generalized Mehler's formula, and motivate the introduction of some particular Heisenberg observables, from a nonlinear point of view.

In Section 4 we state some results about (1.2) in the semi-classical limit; we outline the techniques, and discuss their limitations.

In Sections 5] and 6] we set $\varepsilon=1$, and analyze the influence of the potential $V$ on finite time blow-up and global existence issues.

\section{General Setting And COnsequences}

2.1. Some results on the nonlinear Schrödinger equation. In this paragraph, we assume $\varepsilon=1$, and recall a few things about the Cauchy problem

$$
i \partial_{t} u+\frac{1}{2} \Delta u=\lambda|u|^{2 \sigma} u \quad ;\left.\quad u\right|_{t=0}=u_{0}
$$

where $x \in \mathbb{R}^{n}, \lambda \in \mathbb{R}$, and $\sigma>0$ with $\sigma<\frac{2}{n-2}$ if $n \geq 3$. All the results we mention can be found in 14 .

Theorem 2.1. Suppose $u_{0} \in H^{1}\left(\mathbb{R}^{n}\right)$, and $\sigma>0$ as above. Then there exist $T_{*}, T^{*}>0$, and a unique maximal solution

$$
u \in C(]-T_{*}, T^{*}\left[; H^{1}\right) \cap L_{\mathrm{loc}}^{q}(]-T_{*}, T^{*}\left[; W^{1,2 \sigma+2}\right)
$$

to (2.1), where $q=\frac{4 \sigma+4}{n \sigma}$. It is maximal in the sense that if $T^{*}<\infty$, then

$$
\lim _{t \rightarrow T^{*}}\left\|\nabla_{x} u(t)\right\|_{L^{2}}=+\infty .
$$

In addition, the following quantities are independent of time:

$$
\begin{aligned}
& \text { Mass: }\|u(t)\|_{L^{2}} \equiv\left\|u_{0}\right\|_{L^{2}} \\
& \text { Energy: } E_{0}:=\frac{1}{2}\left\|\nabla_{x} u(t)\right\|_{L^{2}}^{2}+\frac{\lambda}{\sigma+1}\|u(t)\|_{L^{2 \sigma+2}}^{2 \sigma+2}=\text { const. }
\end{aligned}
$$


This result was first proved in 24] (with a slightly different statement), and revisited in [26, [55]. The proof relies on a fixed point argument on Duhamel's formula

$$
u(t)=U_{0}(t) u_{0}-i \lambda \int_{0}^{t} U_{0}(t-s)\left(|u|^{2 \sigma} u\right)(s) d s,
$$

where following the notation introduced in Section $1 U_{0}(t)=e^{i \frac{t}{2} \Delta}$. The modern tool to prove this result is Strichartz estimates, after [51. We recall a statement for the case of Schrödinger equations [55] 34] (see [10] for the following adaptation):

Lemma 2.2. Let $(U(t))_{t \in \mathbb{R}}$ be a unitary group on $L^{2}\left(\mathbb{R}^{n}\right)$, satisfying the dispersive estimate

$$
\|U(t)\|_{L^{1} \rightarrow L^{\infty}} \leq \mathrm{w}(t)^{n / 2}, \quad \text { with } \mathrm{w} \geq 0 \text { and } \mathrm{w} \in L_{w}^{1}(\mathbb{R}) .
$$

We say that a pair $(q, r)$ is admissible if

$$
\frac{2}{q}+\frac{n}{r}=\frac{n}{2} ; \quad q, r \geq 2, \text { with } r<\frac{2 n}{n-2} .
$$

Then for any $T \in \overline{\mathbb{R}}_{+}$, and any admissible pairs $(q, r)$ and $(\widetilde{q}, \widetilde{r})$, we have

$$
\begin{aligned}
\|U(t) f\|_{L^{q}(]-T, T\left[; L^{r}\right)} & \leq\left\|\mathrm{w} \mathbb{1}_{]-2 T, 2 T[}\right\|_{L_{w}^{1}}^{1 / q}\|f\|_{L^{2}}, \\
\left\|\int_{0}^{t} U(t-s) F(s) d s\right\|_{L^{q}(]-T, T\left[; L^{r}\right)} & \leq\left\|\mathrm{w} \mathbb{1}_{]-2 T, 2 T[}\right\|_{L_{w}^{1 / q+1 / \widetilde{q}}}^{1 / F \|_{L^{\tilde{q}^{\prime}}(]-T, T\left[; L^{\tilde{r}^{\prime}}\right)} .}
\end{aligned}
$$

It is straightforward that $U_{0}(t)$ satisfies the assumptions of Lemma 2.2 with $\mathrm{w}(t)=(2 \pi|t|)^{-1}$, from the formula

$$
U_{0}(t) f(x)=\frac{1}{(2 i \pi t)^{n / 2}} \int_{\mathbb{R}^{n}} e^{i \frac{|x-y|^{2}}{2 t}} f(y) d y .
$$

Theorem 2.1] then follows from Strichartz and Hölder's inequalities, as well as Gagliardo-Nirenberg inequalities when $\sigma \geq \frac{2}{n}$. A similar result holds when $u_{0} \in \Sigma$; essentially, we can remember that in addition, $u \in C(\mathbb{R} ; \Sigma)$.

In the $H^{1}$ or $\Sigma$ case, global existence can be deduced from the conservations (2.2), when $\lambda>0$ (defocusing nonlinearity), or $\lambda<0$ and $\sigma<\frac{2}{n}$ for instance. If $\lambda<0$ and $\sigma \geq \frac{2}{n}$, finite time blow-up may occur:

Proposition 2.3 (Virial Theorem, 27]). Assume $\lambda<0$ and $\sigma \geq \frac{2}{n}$ (with $\sigma<\frac{2}{n-2}$ if $n \geq 3$ ). If $u_{0} \in \Sigma$ is such that $E_{0}<0$, then solutions to (2.1) blow up in finite time, in the future and in the past: $T_{*}$ and $T^{*}$ are finite.

The idea of the proof for this result is to introduce the function $y(t)=\|x u(t)\|_{L^{2}}^{2}$ and to notice that under the above assumptions, $\ddot{y}(t) \lesssim E_{0}<0$. Since $y(t) \geq 0$, this proves that a singularity appears, both for $t>0$ and for $t<0$.

A slightly different approach to recover this result is to use the Galilean operator $J(t)=x+i t \nabla_{x}$, and the pseudo-conformal conservation law, discovered in [25]:

$$
\frac{d}{d t}\left(\frac{1}{2}\|J(t) u\|_{L^{2}}^{2}+\frac{\lambda}{\sigma+1} t^{2}\|u(t)\|_{L^{2 \sigma+2}}^{2 \sigma+2}\right)=\frac{\lambda}{\sigma+1}(2-n \sigma) t\|u(t)\|_{L^{2 \sigma+2}}^{2 \sigma+2} .
$$

As noticed in [54, expanding the above formula, one retrieves Proposition 2.3 Another application of the pseudo-conformal conservation law, as motivated in 25], concerns scattering theory:

Proposition 2.4 (Scattering theory in $\Sigma$, 25, 28, 15, 40]). Assume $\frac{2}{n+2}<\sigma<\frac{2}{n-2}$ if $n \geq 2, \sigma>1$ if $n=1$. Assume in addition $\lambda>0$. Denote

$$
\sigma_{0}(n):=\frac{2-n+\sqrt{n^{2}+12 n+4}}{4 n} .
$$


- Let $u_{-} \in \Sigma$. There exists a unique $u \in C(\mathbb{R} ; \Sigma)$ solution of (2.1), such that

$$
\lim _{t \rightarrow-\infty}\left\|U_{0}(-t) u(t)-u_{-}\right\|_{\Sigma}=0 .
$$

- Let $u_{0} \in \Sigma$. If $\sigma \geq \sigma_{0}(n)$ or if $\left\|u_{0}\right\|_{\Sigma}$ is sufficiently small, then there exists a unique $u_{+} \in \Sigma$ such that if $u \in C(\mathbb{R} ; \Sigma)$ is the solution of (2.1), then

$$
\lim _{t \rightarrow+\infty}\left\|U_{0}(-t) u(t)-u_{+}\right\|_{\Sigma}=0 .
$$

We denote by $S: u_{-} \mapsto u_{+}$the scattering operator.

We emphasize two properties of the Galilean operators, from which the above results (Equation (2.6) and Proposition 2.4) follow:

(i) $J(t)$ is an Heisenberg observable:

$$
J(t)=U_{0}(t) x U_{0}(-t) .
$$

(ii) It also reads

$$
J(t)=i t e^{i \frac{|x|^{2}}{2 t}} \nabla_{x}\left(e^{-i \frac{|x|^{2}}{2 t}} .\right) .
$$

As a consequence of (2.7), $J(t)$ commutes with the linear part of (2.1),

$$
\left[J(t), i \partial_{t}+\frac{1}{2} \Delta\right]=0 .
$$

The second point has two interesting straightforward consequences:

(ii)' Weighted Gagliardo-Nirenberg inequalities: for $2 \leq p<\frac{2 n}{n-2}(2 \leq p \leq \infty$ if $n=1$ ), there exists $C_{p}$ depending only on $n$ and $p$ such that

$$
\|f\|_{L^{p}\left(\mathbb{R}^{n}\right)} \leq \frac{C_{p}}{|t|^{\delta(p)}}\|f\|_{L^{2}\left(\mathbb{R}^{n}\right)}^{1-\delta(p)}\|J(t) f\|_{L^{2}\left(\mathbb{R}^{n}\right)}^{\delta(p)} ; \quad \delta(p):=n\left(\frac{1}{2}-\frac{1}{p}\right) .
$$

(ii)" If $F(z)=G\left(|z|^{2}\right) z$ is $C^{1}$, then $J(t)$ acts like a derivative on $F(w)$ :

$$
J(t)(F(w))=\partial_{z} F(w) J(t) w-\partial_{\bar{z}} F(w) \overline{J(t) w} .
$$

Roughly speaking, (2.9) and (2.11) make it possible to have estimates for $J(t) u$ in the same way as for $\nabla_{x} u$, when $u$ solves (2.1). Then (2.10) yields dispersive estimates for the nonlinear equation, which are the same as in the linear case, provided that $J(t) u \in L_{t}^{\infty} L_{x}^{2}$. These arguments are the key ingredients for the first point of Proposition 2.4 (existence of wave operators). Then (2.6) yields estimates on $\|J(t) u\|_{L^{2}}$ which, along with the conservation of mass (2.2), prove the second point of Proposition 2.4 (asymptotic completeness).

Remark. The properties (2.9), (2.10) and (2.11) are the analog of those satisfied by the conformal Killing vector-fields used to study the wave equation (36]).

2.2. Introducing a potential. We now turn to the case of (1.2). Drawing a parallel with the case of (2.1), the first tool we seek is Strichartz estimates for $U_{V}$. This has been, and this is still, a very active area of research. The possible presence of eigenvalues shows that in general, one cannot expect not only the same global dispersion as for $U_{0}$, but also global in time Strichartz estimates. In a more subtle way, resonances may also be an obstacle to dispersion ([30]). Many results have been obtained though, and we refer to the introduction of [48 for a very nice review. In [48, Strichartz estimates are obtained for potentials which may depend of time, a case we do not consider. Notice that these involved results rely on perturbation arguments (see 43] for an interesting exception). We would like to consider the case where the potential may really change the dynamics of the Laplacian, and as introduced in (1.2), we assume that $V \in C^{\infty}\left(\mathbb{R}^{n} ; \mathbb{R}\right)$. 
It is well known that if $V(x) \geq-a|x|^{2}-b$ for some $a, b>0$, then $H_{V}$ is essentially self-adjoint on $C_{0}^{\infty}\left(\mathbb{R}^{n}\right)$, and that this is a sharp result: if $V(x)=-|x|^{4}$ for instance, this property fails (classical trajectories can reach infinite speed, see [46] 20]). On the other hand, if $V$ is positive and grow faster than quadratically, then at least for $n=1$, the kernel of $U_{V}$ is nowhere $C^{1}([56])$, but smoothing properties make it possible to solve the nonlinear problem (1.2) in some cases (57]). We shall restrict our attention to sub-quadratic potentials, for which it is possible to construct a parametrix, locally in time:

Proposition $2.5(22,23)$. Let $V \in C^{\infty}\left(\mathbb{R}^{n} ; \mathbb{R}\right)$ be such that $\partial^{\alpha} V \in L^{\infty}\left(\mathbb{R}^{n}\right)$ for $|\alpha| \geq 2$. Then there exists $\delta>0$ independent of $\varepsilon \in] 0,1]$ such that for $|t| \leq \delta$,

$$
U_{V}^{\varepsilon}(t) f(x)=e^{-i n \frac{\pi}{4} \operatorname{sgn} t} \frac{1}{|2 \pi \varepsilon t|^{n / 2}} \int_{\mathbb{R}^{n}} k^{\varepsilon}(t, x, y) e^{i S(t, x, y) / \varepsilon} f(y) d y,
$$

where $S$ solves the eikonal equation

$$
\partial_{t} S+\frac{1}{2}\left|\nabla_{x} S\right|^{2}+V(x)=0,
$$

and $\partial_{x}^{\alpha} \partial_{y}^{\beta} k^{\varepsilon} \in L^{\infty}(]-\delta, \delta\left[\times \mathbb{R}^{2 n}\right)$ for all $\alpha, \beta \in \mathbb{N}^{n}$, uniformly in $\left.\left.\varepsilon \in\right] 0,1\right]$.

This result relies on perturbation arguments: for small times, the influence of the potential on classical trajectories is controlled, hence a formula similar to (2.5).

Since we know that $U_{V}^{\varepsilon}$ is a unitary group on $L^{2}$, the above result shows that for $|t| \leq \delta, U_{V}^{\varepsilon}$ is dispersive:

$$
\left\|U_{V}^{\varepsilon}(t)\right\|_{L^{1} \rightarrow L^{\infty}} \lesssim \frac{1}{|\varepsilon t|^{-n / 2}}
$$

We infer that there are local in time Strichartz estimates. Notice that in general, one cannot expect global in time estimates: when $V$ is the harmonic potential or is lattice periodic, $H_{V}$ (hence $H_{V}^{\varepsilon}$ ) has eigenvalues.

One can then mimic the proof of Theorem 2.1. Unlike for the case of (2.1), the gradient does not commute with the linear part of the equation:

$$
\left(i \varepsilon \partial_{t}+\frac{1}{2} \varepsilon^{2} \Delta-V(x)\right) \nabla_{x} u^{\varepsilon}=u^{\varepsilon} \nabla_{x} V+\lambda^{\varepsilon} \nabla_{x}\left(\left|u^{\varepsilon}\right|^{2 \sigma} u^{\varepsilon}\right) .
$$

The new term is $u^{\varepsilon} \nabla_{x} V$. Since $V$ is sub-quadratic, $|\nabla V(x)| \lesssim 1+|x|$, which suggests to consider $x u^{\varepsilon}$ as a third unknown, after $u^{\varepsilon}$ and $\nabla_{x} u^{\varepsilon}$. We then have

$$
\left(i \varepsilon \partial_{t}+\frac{1}{2} \varepsilon^{2} \Delta-V(x)\right) x u^{\varepsilon}=\nabla_{x} u^{\varepsilon}+\lambda^{\varepsilon} x\left(\left|u^{\varepsilon}\right|^{2 \sigma} u^{\varepsilon}\right) .
$$

It is easy to prove:

Lemma 2.6. Suppose that $V \in C^{\infty}\left(\mathbb{R}^{n} ; \mathbb{R}\right)$ is sub-quadratic, $u_{0}^{\varepsilon} \in \Sigma, \lambda^{\varepsilon} \in \mathbb{R}$, and $\sigma>0$ with $\sigma<\frac{2}{n-2}$ if $n \geq 3$. Then there exist $T^{\varepsilon}>0$ (depending on $n, \sigma, V,\left|\lambda^{\varepsilon}\right|$ and $\left.\left\|u_{0}^{\varepsilon}\right\|_{\Sigma}\right)$, and a unique solution

$$
u^{\varepsilon} \in C(]-T^{\varepsilon}, T^{\varepsilon}[; \Sigma) \cap L_{\text {loc }}^{q}(]-T^{\varepsilon}, T^{\varepsilon}\left[; W^{1,2 \sigma+2}\right)
$$

to (1.2), where $q=\frac{4 \sigma+4}{n \sigma}$. The following quantities are independent of time:

Mass: $\left\|u^{\varepsilon}(t)\right\|_{L^{2}} \equiv\left\|u_{0}^{\varepsilon}\right\|_{L^{2}}$,

Energy: $E_{V}^{\varepsilon}:=\frac{1}{2}\left\|\varepsilon \nabla_{x} u^{\varepsilon}(t)\right\|_{L^{2}}^{2}+\frac{\lambda^{\varepsilon}}{\sigma+1}\left\|u^{\varepsilon}(t)\right\|_{L^{2 \sigma+2}}^{2 \sigma+2}+\int_{\mathbb{R}^{n}} V(x)\left|u^{\varepsilon}(t, x)\right|^{2} d x$.

In addition, if $\sigma<\frac{2}{n}\left(L^{2}\right.$ sub-critical case), then one can take $T^{\varepsilon}=+\infty$. 
Notice that without further assumption on $V$, we cannot state a criterion for the obstruction to global existence, as in Theorem 2.1. Global existence in the $L^{2}$ sub-critical case follows from the same ideas as in 53: one has global existence at the $L^{2}$ level, from Strichartz inequalities (local in time inequalities are sufficient) and the conservation of mass. One deduces global existence in $\Sigma$ by considering $\left|u^{\varepsilon}\right|^{2 \sigma}$ as a potential in the equations for $\nabla_{x} u^{\varepsilon}$ and $x u^{\varepsilon}$.

If in addition $V$ is non-negative, then the assumption $u_{0}^{\varepsilon} \in \Sigma$ can be weakened: it suffices to consider initial data in the domain of $\sqrt{-\Delta+V}$ (see [44, 14]). In that case, the obstruction to global existence is the same as in Theorem [2.1]

2.3. Removing the potential. It turns out that for some specific potentials, an explicit change of variables makes it possible to relate the solutions of (1.2) to the solutions of the same equation with $V \equiv 0$. These potentials are:

- The linear potential (Stark potential): $V(x)=E \cdot x$.

- The isotropic harmonic potential $V(x)=|x|^{2}$, in the case where the nonlinearity is $L^{2}$-critical, $\sigma=\frac{2}{n}$.

- The isotropic repulsive harmonic potential $V(x)=-|x|^{2}$, in the case where the nonlinearity is $L^{2}$-critical.

Introduce $v^{\varepsilon}$ the solution to the equation with no potential:

$$
i \varepsilon \partial_{t} v^{\varepsilon}+\frac{1}{2} \varepsilon^{2} \Delta v^{\varepsilon}=\lambda^{\varepsilon}\left|v^{\varepsilon}\right|^{2 \sigma} v^{\varepsilon} \quad ;\left.\quad v^{\varepsilon}\right|_{t=0}=u_{0}^{\varepsilon}\left(=\left.u^{\varepsilon}\right|_{t=0}\right)
$$

2.3.1. Linear potential. Assume that $V(x)=E \cdot x$, where $E \in \mathbb{R}^{n}$ is constant. Then as noticed in 13, the Avron-Herbst formula, discovered in the linear case $\lambda^{\varepsilon}=0$ (1]), relates $u^{\varepsilon}$ and $v^{\varepsilon}$ :

$$
u^{\varepsilon}(t, x)=v^{\varepsilon}\left(t, x+\frac{t^{2}}{2} E\right) e^{-i\left(t E \cdot x+\frac{t^{3}}{6}|E|^{2}\right) / \varepsilon} .
$$

Therefore, one can use Theorem 2.1 to deduce local existence results in $H^{1}$ and see that the only obstruction to global existence is the unboundedness of $\nabla_{x} u^{\varepsilon}$ in $L^{2}$. Similarly, the Stark potential does not change the possible blow-up time, nor the scattering theory (replace $U_{0}$ by $U_{V}$ in Proposition 2.4 . In the case of finite time blow-up, the linear potential only shifts the set where this phenomenon occurs: if $v^{\varepsilon}$ blows up on a set $X$ at time $T^{\varepsilon}>0$ (see e.g. 38 for a definition of such a set), then $u^{\varepsilon}$ blows up (at time $T^{\varepsilon}$ ) on $X-\frac{\left(T^{\varepsilon}\right)^{2}}{2} E$.

2.3.2. Isotropic harmonic potential and conformal nonlinearity. Assume that $\sigma=\frac{2}{n}$ and $V(x)=\frac{\omega^{2}}{2}|x|^{2}$ with $\omega>0$. Then provided that the right hand side is defined,

$$
u^{\varepsilon}(t, x)=\frac{1}{(\cos \omega t)^{n / 2}} e^{-i \frac{\omega}{2 \varepsilon} x^{2} \tan \omega t} v^{\varepsilon}\left(\frac{\tan \omega t}{\omega}, \frac{x}{\cos \omega t}\right) .
$$

This was first noticed in [41] for the linear case $\left(\lambda^{\varepsilon}=0\right)$, and in [49, 6] for the nonlinear case with critical nonlinearity. Note that despite this expression, $u^{\varepsilon}$ needs not blow-up at time $t_{1}=\frac{\pi}{2 \omega}$ (when the cosine is zero). Indeed, as $t \rightarrow t_{1}$, the time variable $\frac{\tan \omega t}{\omega}$ for $v^{\varepsilon}$ goes to infinity. Then dispersive properties of solutions to (2.1) may compensate the cancellation of the cosine.

However, it is true that the harmonic potential generates more blowing-up solutions (the criterion for finite time blow-up is the same as in Theorem 2.11). First, if $v^{\varepsilon}$ blows up at time $T^{\varepsilon}>0$, then $u^{\varepsilon}$ blows up at time $\frac{\arctan \left(\omega T^{\varepsilon}\right)}{T^{\varepsilon}}$, which is always smaller than $T^{\varepsilon}$. Second, if $v^{\varepsilon}$ is not a dispersive solution, typically of solitary wave $v^{\varepsilon}(t, x)=e^{i \kappa t / \varepsilon} Q\left(\frac{x}{\varepsilon}\right)$, then $u^{\varepsilon}$ blows up at time $\frac{\pi}{2 \omega}$. From a heuristic point of view, the confining properties of the harmonic potential are sufficient to concentrate an energy which is not naturally dispersed. 
Similarly, because the harmonic potential prevents the solutions from being dispersive as in the case $V \equiv 0$, no scattering theory must be expected.

2.3.3. Isotropic repulsive harmonic potential and conformal nonlinearity. Assume that $\sigma=\frac{2}{n}$ and $V(x)=-\frac{\omega^{2}}{2}|x|^{2}$ with $\omega>0$. Then as noticed in $[$,

$$
u^{\varepsilon}(t, x)=\frac{1}{(\cosh (\omega t))^{n / 2}} e^{i \frac{\omega}{2 \varepsilon}|x|^{2} \tanh (\omega t)} v^{\varepsilon}\left(\frac{\tanh (\omega t)}{\omega}, \frac{x}{\cosh (\omega t)}\right) .
$$

A criterion for global existence is not obvious in this case, since the potential is unbounded from below. It was proved in 8 that for an isotropic repulsive harmonic potential (and a general nonlinearity as in (1.2)), the obstruction to global existence is the same as in Theorem 2.1 (see also Section [5). Opposite to the harmonic potential mentioned above, the repulsive harmonic potential tends to prevent blowup. If $v^{\varepsilon}$ blows up at time $T^{\varepsilon}>0$, then $u^{\varepsilon}$ blows up at time $\frac{\arg \tanh \left(\omega T^{\varepsilon}\right)}{\omega}$ if $\omega T^{\varepsilon}<1$. That means that for "small" values of $\omega$, blow-up is delayed. On the other hand, if $\omega T^{\varepsilon} \geq 1$, then $u^{\varepsilon}$ does not "see" the blow up of $v^{\varepsilon}$, and is global. If $\omega T^{\varepsilon}>1, u^{\varepsilon}$ has even exponential decay as $t \rightarrow+\infty$. The limiting case $\omega T^{\varepsilon}=1$ is interesting: it is known that there exists no $\left(L^{2}\right)$ solitary wave (31, 33, 39]). The analog of a solitary wave is a solution that grows exponentially in time (see $[8]$ ).

Unlike the harmonic potential, the repulsive harmonic potential tends to spread out a solution to (2.1): we saw how it changes the finite time blow-up phenomenon. It is not surprising that things go very well as far as scattering is concerned, thanks to exponential decay. We study this issue more precisely in Section [6]

\section{MEHLER'S FORMULA AND APPLICATIONS}

When $V$ is a second order polynomial, a lot can be said. First, the linear case is extremely favorable, because $U_{V}^{\varepsilon}$ is known explicitly: this is the (generalized) Mehler's formula (21, 29]). If $V(x)=\sum a_{j k} x_{j} x_{k}+\sum b_{j} x_{j}+c$, then reducing the quadratic part, a change of orthonormal basis (which leaves the Laplace operator invariant) and a change of the origin simplify the expression of $V$ :

$$
V(x)=\sum_{j=1}^{n}\left(\delta_{j} \frac{\omega_{j}^{2}}{2} x_{j}^{2}+\widetilde{b}_{j} x_{j}\right)+\widetilde{c} ; \delta_{j} \in\{-1,0,1\}, \omega_{j}>0, \delta_{j} \widetilde{b}_{j}=0 .
$$

Using Avron-Herbst formula (2.14), we can get rid of linear terms $\widetilde{b}_{j} x_{j}$. Taking $e^{i c t} / \varepsilon u^{\varepsilon}$ as a new unknown function also removes the constant term. We therefore assume that $V$ is of the form

$$
V(x)=\sum_{j=1}^{n} \delta_{j} \frac{\omega_{j}^{2}}{2} x_{j}^{2} \quad ; \quad \delta_{j} \in\{-1,0,1\}, \omega_{j}>0 .
$$

The Mehler's formula then reads:

$$
U_{V}^{\varepsilon}(t) f(x)=\prod_{j=1}^{n}\left(\frac{1}{2 i \pi \varepsilon g_{j}(t)}\right)^{1 / 2} \int_{\mathbb{R}^{n}} e^{i S(t, x, y) / \varepsilon} f(y) d y,
$$

where

$$
S(t, x, y)=\sum_{j=1}^{n} \frac{1}{g_{j}(t)}\left(\frac{x_{j}^{2}+y_{j}^{2}}{2} h_{j}(t)-x_{j} y_{j}\right)
$$


and the functions $g_{j}$ and $h_{j}$, related to the classical trajectories, are given by:

$$
\left(g_{j}(t), h_{j}(t)\right)=\left\{\begin{aligned}
\left(\frac{\sinh \left(\omega_{j} t\right)}{\omega_{j}}, \cosh \left(\omega_{j} t\right)\right), & \text { if } \delta_{j}=-1, \\
(t, 1), & \text { if } \delta_{j}=0, \\
\left(\frac{\sin \left(\omega_{j} t\right)}{\omega_{j}}, \cos \left(\omega_{j} t\right)\right), & \text { if } \delta_{j}=+1 .
\end{aligned}\right.
$$

Recall that if there exists $\delta_{j}=+1$, then $e^{-i t H_{V}^{\varepsilon}}$ has some singularities, periodically in time (see e.g. 32]). This affects the above formula with phase factors we did not write (which can be incorporated in the definition of $\left(i g_{j}(t)\right)^{1 / 2}$ ).

Let us examine the Strichartz estimates we can deduce. If $\delta_{j}=+1$ for any $j$, then only local in time Strichartz estimates are available. This is not surprising, since the harmonic oscillator has eigenvalues. On the other hand, if $\omega_{j}=-1$ for any $j$, then we have $\left\|U_{V}^{\varepsilon}(t)\right\|_{L^{1} \rightarrow L^{\infty}} \leq|2 \pi \varepsilon t|^{-n / 2}$, an estimate which is independent of all the $\omega_{j}$ 's: we have the same dispersion as in the case with no potential (recall from the previous section that the repulsive harmonic potential accelerates the "particle"), and global in time Strichartz estimates follow. Actually, this property remains if $\delta_{j}=-1$ for at least one $j$. This stems from the formulation of Lemma 2.2 the function $\mathrm{w}(t)$ given by Mehler's formula is in the weak $L^{1}$ space for small values of $|t|$, and in the strong $L^{1}$ space for "large" values of $|t|$.

Remark. We would like to point out that in the case where $\delta_{j}=-1$ for at least one $j$, we have global in time Strichartz estimates, while there are trapped trajectories. The one-dimensional case shows the mechanism. If $n=1$ and $\omega=1$, the classical symbol of $H_{V}^{\varepsilon}$ is $\frac{1}{2}\left(\xi^{2}-x^{2}\right)$. Computing the Hamilton flow, we have

$$
x(t)=x_{0} \cosh t+\xi_{0} \sinh t=\frac{x_{0}+\xi_{0}}{2} e^{t}+\frac{x_{0}-\xi_{0}}{2} e^{-t} .
$$

If $x_{0}+\xi_{0}=0$, then the trajectory is trapped in the future. This aspect can be compared to the results of [16, 18, where it is proved that smoothing effects occur in the future provided that the classical trajectories are not trapped in the past. However, the results of 16 include potentials which grow at most linearly in $x$, and [18, does not consider the case of potentials. On the other hand, smoothing effects yield another approach to prove Strichartz estimates (see e.g. [50]). In our case, there are trajectories trapped in the past or in the future, but global in time Strichartz estimates are available. It seems that the link between classical trajectories and (global in time) Strichartz estimates remains to be clarified.

To introduce the tools we use in the rest of the analysis, recall the approach followed in 9 . We consider the case of an isotropic harmonic potential, $V(x)=$ $\frac{\omega^{2}}{2}|x|^{2}$. In the linear case $\lambda^{\varepsilon}=0, u^{\varepsilon}$ is therefore given by Mehler's formula. A formal stationary argument (which can be justified) shows that if $u_{0}^{\varepsilon}=f$ does not depend on $\varepsilon$, then for $|t|<\frac{\pi}{2 \omega}$,

$$
u^{\varepsilon}(t, x) \underset{\varepsilon \rightarrow 0}{\sim} \frac{1}{(\cos (\omega t))^{n / 2}} f\left(\frac{x}{\cos (\omega t)}\right) e^{-i \omega \frac{|x|^{2}}{2 \varepsilon}} \tan (\omega t)=: u_{\text {app }}^{\varepsilon}(t, x) .
$$

In the nonlinear case, we expect that if, say $\lambda^{\varepsilon}=\varnothing\left(\varepsilon^{k}\right)$ with $k$ sufficiently large, then nonlinear effects should be negligible in the semi-classical limit, at least before the first singularity at time $\frac{\pi}{2 \omega}$. This is proved in [9], and we recall the argument in Section 4 A natural candidate for an approximate solution is then given by $u_{\mathrm{app}}^{\varepsilon}$. To prove the approximation is valid in the nonlinear case, $L^{2}$ estimates are not sufficient: $L^{p}$ estimates are needed, for other values of $p$, and one can think of Gagliardo-Nirenberg inequalities, $\|g\|_{L^{p}} \lesssim\|g\|_{L^{2}}^{1-\delta(p)}\|\nabla g\|_{L^{2}}^{\delta(p)}$. 
However, these inequalities yield $\left\|u_{\text {app }}^{\varepsilon}(t)\right\|_{L^{p}} \lesssim \varepsilon^{-\delta(p)}$, which is terrible in the limit $\varepsilon \rightarrow 0$ for $|t|<\frac{\pi}{2 \omega}$. This "bad" power of $\varepsilon$ stems from the highly oscillatory phase. Note that the nonlinearity we consider, $F(z)=|z|^{2 \sigma} z$, does not create new harmonics in a single phase WKB asymptotics, if only one harmonic is present initially. Therefore, in our case, there is only one phase and one harmonic to take care about. This suggests to replace the gradient in Gagliardo-Nirenberg inequalities by the operator $\nabla_{x}\left(e^{i \omega \frac{|x|^{2}}{2 \varepsilon} \tan (\omega t)}.\right)$. In view of the expression for $u_{\mathrm{app}}^{\varepsilon}$, we introduce more precisely:

$J^{\varepsilon}(t)=i \cos (\omega t) e^{-i \omega \frac{|x|^{2}}{2 \varepsilon} \tan (\omega t)} \nabla_{x}\left(e^{i \omega \frac{|x|^{2}}{2 \varepsilon} \tan (\omega t)} \cdot\right)=-\omega^{2} \sin (\omega t) \frac{x}{\varepsilon}+i \cos (\omega t) \nabla_{x}$.

Introduce the operator

$$
H^{\varepsilon}(t)=\cos (\omega t) x+i \frac{\sin (\omega t)}{\omega} \varepsilon \nabla_{x} .
$$

We can then rewrite the energy $E_{V}^{\varepsilon}$ (which is constant from Lemma 2.6), as

$$
E_{V}^{\varepsilon}=\frac{1}{2}\left\|\varepsilon J^{\varepsilon}(t) u^{\varepsilon}\right\|_{L^{2}}^{2}+\frac{\omega^{2}}{2}\left\|H^{\varepsilon}(t) u^{\varepsilon}\right\|_{L^{2}}^{2}+\frac{\lambda^{\varepsilon}}{\sigma+1}\left\|u^{\varepsilon}(t)\right\|_{L^{2 \sigma+2}}^{2 \sigma+2} .
$$

More generally, if the potential if of the form (3.1), we define:

$$
\left(\begin{array}{c}
J_{j}(t) \\
H_{j}(t)
\end{array}\right)=\left(\begin{array}{cc}
-\delta_{j} \omega_{j}^{2} g_{j}(t) & h_{j}(t) / \varepsilon \\
\varepsilon h_{j}(t) & g_{j}(t)
\end{array}\right)\left(\begin{array}{c}
x_{j} / \varepsilon \\
i \varepsilon \partial_{j}
\end{array}\right), \quad \forall j \geq 1 .
$$

We set $J^{\varepsilon}=\left(J_{j}^{\varepsilon}\right)_{1 \leq j \leq n}$ and $H^{\varepsilon}=\left(H_{j}^{\varepsilon}\right)_{1 \leq j \leq n}$. These operators have been known for years, in the linear theory (see e.g. [52, 47]). They are Heisenberg observables:

$$
J^{\varepsilon}(t)=U_{V}^{\varepsilon}(t) i \nabla_{x} U_{V}^{\varepsilon}(-t) \quad ; \quad H^{\varepsilon}(t)=U_{V}^{\varepsilon}(t) x U_{V}^{\varepsilon}(-t) .
$$

The fact that such Heisenberg observables can be computed exactly is due to the assumption that the potential is a second order polynomial. Note that if $\delta_{j}=0$, then we recover the two operators introduced in Section 2.1 the gradient and the Galilean operator. We will see in the next section that when $V$ is of the form (3.1), then $J^{\varepsilon}$ and $H^{\varepsilon}$ satisfy the same properties as those emphasized in Section 2.1

\section{Semi-Classical analysis in a NONLINEAR FRAmeWORK}

The main features of the operators $J^{\varepsilon}$ and $H^{\varepsilon}$ are the following:

Lemma 4.1. Let $V$ be of the form (3.1). The operators $J^{e}$ and $H^{\varepsilon}$ satisfy:

(i) They commute with the linear part of (1.2):

$$
\left[J^{\varepsilon}(t), i \varepsilon \partial_{t}+\frac{1}{2} \varepsilon^{2} \Delta-V(x)\right]=\left[H^{\varepsilon}(t), i \varepsilon \partial_{t}+\frac{1}{2} \varepsilon^{2} \Delta-V(x)\right]=0 .
$$

(ii) They can be written as

$$
\begin{aligned}
J_{j}^{\varepsilon}(t) & =i h_{j}(t) e^{i \phi_{1}(t, x) / \varepsilon} \partial_{j}\left(e^{-i \phi_{1}(t, x) / \varepsilon} \cdot\right), \\
H_{j}^{\varepsilon}(t) & =i g_{j}(t) e^{i \phi_{2}(t, x) / \varepsilon} \partial_{j}\left(e^{-i \phi_{2}(t, x) / \varepsilon} \cdot\right),
\end{aligned}
$$

for some real-valued phases $\phi_{1}$ and $\phi_{2}$, solutions of the eikonal equation (2.13).

$(\text { ii })^{\prime}$ They yield weighted Gagliardo-Nirenberg inequalities, such as

$$
\|f\|_{L^{p}} \lesssim\left(\prod_{j=1}^{n}\left|h_{j}(t)\right|^{\delta(p) / n}\right)\|f\|_{L^{2}}^{1-\delta(p)}\|J(t) f\|_{L^{2}}^{\delta(p)} .
$$

(ii)" If $F(z)=G\left(|z|^{2}\right) z$ is $C^{1}$, then $J(t) F(w)$ and $H(t) F(w)$ are given by (2.11). 
The first point is trivial, since $J^{\varepsilon}$ and $H^{e}$ are Heisenberg observables. The last two points follow from the second one, which is the way we found the operator $J^{\varepsilon}$ in the case of the isotropic harmonic potential. We can consider that two algebraic miracles occur: first, we can compute explicitly some interesting Heisenberg observables. Second, these operators can be written like (ii), which is a nice property in view of nonlinear problems. We discuss these aspects further into details below.

We now come to the issue of critical scales in the semi-classical analysis of (1.2). We describe two cases:

- $V$ is an isotropic harmonic potential, and $u_{0}^{\varepsilon}=f$ does not depend on $\varepsilon$.

- $V$ is of the form (3.1) and $u_{0}^{\varepsilon}$ is a concentrating profile.

The first case corresponds to the one that led us to introduce the operators $J^{\varepsilon}$ and $H^{\varepsilon}$. In the linear case, due to the harmonic potential, the solution focuses at the origin at time $t=\frac{\pi}{2 \omega}$. More precisely, the geometry of the propagation in the limit $\varepsilon \rightarrow 0$ is given by the Hamilton flow. The classical Hamiltonian in this case is $p(t, x, \tau, \xi)=\frac{1}{2}\left(\left|\xi^{2}\right|+\omega^{2}|x|^{2}\right)$. The classical trajectories (rays of geometric optics) are given by

$$
x(t)=x_{0} \cos (\omega t)+\xi_{0} \frac{\sin (\omega t)}{\omega} .
$$

Since $f$ does not depend on $\varepsilon$, there is no initial oscillation: $\xi_{0}=0$, and rays meet at the origin, periodically in time. When $\lambda^{\varepsilon}=0$, one has the sharp estimate:

$$
\left\|u^{\varepsilon}(t)\right\|_{L^{p}} \lesssim\left(\frac{1}{|\cos (\omega t)|+\varepsilon|\sin (\omega t)|}\right)^{\delta(p)}
$$

This follows easily from Lemma 4.1 and the conservation of the $L^{2}$ for solution to linear Schrödinger equations. Now assume that $\lambda^{\varepsilon}=\varepsilon^{\alpha}$, for some $\alpha>0$. Then the conservations of mass and energy (Lemma 2.6) show that the solution $u^{\varepsilon}$ is global in time. For $\alpha$ large, the linear solution is expected to be a good approximation for the nonlinear solution. More precisely, two régimes must be considered. Before focusing, the solution is of order $\varnothing(1)$, and one can apply WKB methods. This leads to a linear approximation if $\alpha>1$, nonlinear otherwise. Next, at the focus, the linear solution is of order $\varepsilon^{-n / 2}$, more precisely, we infer from (3.2):

$$
u_{\operatorname{lin}}^{\varepsilon}\left(\frac{\pi}{2 \omega}, x\right) \underset{\varepsilon \rightarrow 0}{\sim}\left(\frac{\omega}{\varepsilon}\right)^{n / 2} \widehat{f}\left(\frac{\omega x}{\varepsilon}\right), \text { where } \widehat{f}(\xi)=\int_{\mathbb{R}^{n}} e^{-i x \cdot \xi} f(x) d x .
$$

The nonlinear term can be viewed as a potential $\varepsilon^{\alpha}\left|u^{\varepsilon}\right|^{2 \sigma}$. Plugging the above asymptotics suggests that a critical value for $\alpha$ at the focus is $\alpha=n \sigma$.

With the arguments given in [9], it is possible to prove that if $\alpha>\max (1, n \sigma)$, then the nonlinearity is negligible in the limit $\varepsilon \rightarrow 0$, locally uniformly in time. The case $\alpha=n \sigma>1$ is shown to be critical:

Theorem $4.2(9])$. Let $V(x)=\frac{|x|^{2}}{2}$, and $2<r<\frac{2 n}{n-2}$, with $r=\infty$ if $n=1$. Assume that the nonlinearity $z \mapsto|z|^{2 \sigma} z$ is twice differentiable, and that $\lambda^{\varepsilon}=\varepsilon^{n \sigma}$, with $n \sigma>1$. Assume moreover that $\sigma>\sigma_{0}(n)$. Then for $k \in \mathbb{N}$, the asymptotics of $u^{\varepsilon}$ for $\frac{\pi}{2}+(k-1) \pi<t<\frac{\pi}{2}+k \pi$ is given, in $L^{2} \cap L^{r}$, by:

$$
u^{\varepsilon}(t, x) \underset{\varepsilon \rightarrow 0}{\sim} \frac{e^{i n \frac{\pi}{4}-i n k \frac{\pi}{2}}}{(2 \pi|\cos t|)^{n / 2}} \widehat{S^{k} \psi_{-}}\left(\frac{-x}{\cos t}\right) e^{-i \frac{|x|^{2}}{2 \varepsilon} \tan t},
$$

where $S^{k}$ denotes the $k$-th iterate of $S$ (which is well defined under our assumptions on $f)$, and $\psi_{-}(x):=(2 i \pi)^{-n / 2} \widehat{f}(x)$.

Note that the phase shift $-n \frac{\pi}{2}$, appearing at each focus crossing, is a linear phenomenon (Maslov-Keller index, see [19]). Thus the only nonlinear effects at leading order occur at the focuses, and are described, in average, by the scattering 
operator $S$ associated to (2.1). We give the main ideas of the proof. The first step consists in proving that before the first focus at time $t=\frac{\pi}{2}$, the function $u_{\text {app }}^{\varepsilon}$ defined by (3.4) is a good approximation for the nonlinear solution $u^{\varepsilon}$. This is equivalent to justifying a WKB asymptotics at leading order, and relies on the sharp estimate given by the operator $J^{\varepsilon}$ and the weighted Gagliardo-Nirenberg inequality it provides (point (ii)' in Lemma 4.1). We can prove that $u_{\text {app }}^{\varepsilon}$ remains close to $u^{\varepsilon}$ (in a space which is essentially $\Sigma$ ), up to time $\frac{\pi}{2}-\Lambda \varepsilon$, in the limit $\Lambda \rightarrow+\infty$, that is, until focusing effects become relevant at leading order. This shows that as predicted, the assumption $\alpha>1$ makes the nonlinearity negligible outside the focus.

When $t-\frac{\pi}{2}=\varnothing(\varepsilon), u^{\varepsilon}$ tends to concentrate near the origin, at scale $\varepsilon ; V(x) u^{\varepsilon}$ becomes negligible, while the nonlinear potential $\varepsilon^{n \sigma}\left|u^{\varepsilon}\right|^{2 \sigma}$ is of order $\varnothing(1)$. This can be proved thanks essentially to the operator $H^{\varepsilon}$. Note that for $t-\frac{\pi}{2}=\varnothing(\varepsilon)$, the operator $J^{\varepsilon}$ and $H^{\varepsilon}$ can be replaced respectively by $\frac{x}{\varepsilon}+i\left(t-\frac{\pi}{2}\right) \nabla_{x}$ and $\varepsilon \nabla_{x}$, which is another way to check that the potential is negligible. The scaling

$$
u^{\varepsilon}(t, x)=\frac{1}{\varepsilon^{n / 2}} \psi^{\varepsilon}\left(\frac{t-\frac{\pi}{2}}{\varepsilon}, \frac{x}{\varepsilon}\right)
$$

turns the description of the caustic crossing into a continuity issue for a scattering problem. We have $\left\|U_{0}(-t)\left(\psi^{\varepsilon}(t)-\psi(t)\right)\right\|_{L^{\infty}(\mathbb{R} ; \Sigma)}=o(1)$, where $\psi$ solves (2.1), with $\psi_{-}$as a Cauchy datum at $t=-\infty$. This scattering state stems from the transition between the two régimes for $u^{\varepsilon}$, which occurs for $t=\frac{\pi}{2}-\Lambda \varepsilon$, in the limit $\Lambda \rightarrow+\infty$.

Next, $u^{\varepsilon}$ leaves the focus in a way described by the second point of Proposition 2.4 and (4.1). The analysis is symmetric to the one before the focus; we prove the asymptotics until $t=\pi$, where the situation is similar to that at time $t=0$. Iterating the analysis yields Theorem 4.2

Remark. With no additional effort, one can treat the case of initial plane oscillations. Let $\xi_{0} \in \mathbb{R}^{n}$, and introduce

$$
\mathrm{u}^{\varepsilon}(t, x)=u^{\varepsilon}\left(t, x-\xi_{0} \sin t\right) e^{i\left(x-\frac{\xi_{0}}{2} \sin t\right) \cdot \xi_{0} \cos t / \varepsilon} .
$$

If $u^{\varepsilon}$ solves (1.2) with $V(x)=\frac{|x|^{2}}{2}$ and $u_{0}^{\varepsilon}=f$, then $\mathrm{u}^{\varepsilon}$ solves (1.2) with initial data $\mathrm{u}^{\varepsilon}{ }_{\mid t=0}=f(x) e^{i \frac{x \cdot \xi_{0}}{\varepsilon}}$.

The second case we analyze is when $V$ is of the general form (3.1) and

$$
u_{0}^{\varepsilon}(x)=\frac{1}{\varepsilon^{n / 2}} \varphi\left(\frac{x}{\varepsilon}\right) \quad(\varphi \in \Sigma) \quad ; \quad \lambda^{\varepsilon}=\varepsilon^{n \sigma} .
$$

If $\lambda^{\varepsilon}=\varepsilon^{\alpha}$ with $\alpha>\max (1, n \sigma)$, then one can show that the evolution of $u^{\varepsilon}$ is linear, at leading order. The case $\lambda^{\varepsilon}=-\varepsilon^{n \sigma}$ has been studied by several authors (see e.g. 3, 35]), in the case $\sigma<\frac{2}{n}$ and $\varphi(x)=R\left(x-x_{0}\right) e^{i x \cdot \xi_{0}}$, where $R$ is a ground state. In that situation, $u^{\varepsilon}$ evolves as a concentrating profile, with profile $R$, along the Hamilton flow associated to $H_{V}$ with data $\left(x_{0}, \xi_{0}\right)$. Heuristically, this is so because there is a balance between the dispersive effects associated to $H_{V}^{\varepsilon}$, and the nonlinear effects (in the case $\lambda^{\varepsilon}<0$, the nonlinearity is attractive).

In the case $\lambda^{\varepsilon}=+\varepsilon^{n \sigma}$, the two effects mentioned above tend to cumulate, and dispersion alters the shape of $u^{\varepsilon}$. Note that even though $\lambda^{\varepsilon}>0$, global existence for $u^{\varepsilon}$ is not obvious, since $V$ is not necessarily signed.

Theorem 4.3 (12]). Let $V$ of the form (3.1), and $u_{0}^{\varepsilon}$ given by (4.2). Assume either that there exists $j$ such that $\delta_{j} \neq 1$, or that $\delta_{j}=1$ for all $j$ and the $\omega_{j}$ 's are not pairwise rationally dependent. Suppose that the nonlinearity $z \mapsto|z|^{2 \sigma} z$ is twice differentiable. Then the following holds.

1. For any $T>0$, there exists $\varepsilon(T)>0$ such that for $0<\varepsilon \leq \varepsilon(T)$, (1.2 has a 
unique solution $u^{\varepsilon} \in C([-T, T] ; \Sigma)$.

2. This solution satisfies the following asymptotics.

- For any $\Lambda>0$,

$$
\begin{aligned}
\limsup _{\varepsilon \rightarrow 0} & \sup _{|t| \leq \Lambda \varepsilon}\left(\left\|u^{\varepsilon}(t)-v^{\varepsilon}(t)\right\|_{L^{2}}+\left\|\varepsilon \nabla_{x} u^{\varepsilon}(t)-\varepsilon \nabla_{x} v^{\varepsilon}(t)\right\|_{L^{2}}\right. \\
& \left.+\left\|\frac{x}{\varepsilon} u^{\varepsilon}(t)-\frac{x}{\varepsilon} v^{\varepsilon}(t)\right\|_{L^{2}}\right)=0, \quad \text { where } v^{\varepsilon}(t, x)=\frac{1}{\varepsilon^{n / 2}} \psi\left(\frac{t}{\varepsilon}, \frac{x}{\varepsilon}\right),
\end{aligned}
$$

and $\psi \in C(\mathbb{R} ; \Sigma)$ is the solution to (2.1) such that $\psi_{\mid t=0}=\varphi$.

- Beyond this boundary layer, we have

$$
\begin{gathered}
\limsup _{\varepsilon \rightarrow 0} \sup _{\Lambda \varepsilon \leq \pm t \leq T}\left(\left\|u^{\varepsilon}(t)-u_{ \pm}^{\varepsilon}(t)\right\|_{L^{2}}+\left\|\varepsilon \nabla_{x} u^{\varepsilon}(t)-\varepsilon \nabla_{x} u_{ \pm}^{\varepsilon}(t)\right\|_{L^{2}}\right. \\
\left.+\left\|x u^{\varepsilon}(t)-x u_{ \pm}^{\varepsilon}(t)\right\|_{L^{2}}\right)_{\Lambda \rightarrow+\infty} 0
\end{gathered}
$$

where $u_{ \pm}^{\varepsilon} \in C(\mathbb{R} ; \Sigma)$ are the solutions to

$$
i \varepsilon \partial_{t} u_{ \pm}^{\varepsilon}+\frac{1}{2} \varepsilon^{2} \Delta u_{ \pm}^{\varepsilon}=V(x) u_{ \pm}^{\varepsilon} \quad ; \quad u_{ \pm \mid t=0}^{\varepsilon}=\frac{1}{\varepsilon^{n / 2}} \psi_{ \pm}\left(\frac{x}{\varepsilon}\right),
$$

and $\psi_{ \pm}$are given by Proposition 2.4

This result, as well as the analysis in [9], can be viewed as a nonlinear analog to a result due to F. Nier. In [42], the author studies the problem

$$
i \varepsilon \partial_{t} u^{\varepsilon}+\frac{1}{2} \varepsilon^{2} \Delta u^{\varepsilon}=V(x) u^{\varepsilon}+U\left(\frac{x}{\varepsilon}\right) u^{\varepsilon} \quad ; \quad u_{\mid t=0}^{\varepsilon}=\frac{1}{\varepsilon^{n / 2}} \varphi\left(\frac{x}{\varepsilon}\right),
$$

where $U$ is a short range potential. The potential $V$ in that case is bounded as well as all its derivatives. Under suitable assumptions, the influence of $U$ occurs near $t=0$ and is localized near the origin, while only the value $V(0)$ of $V$ at the origin is relevant in this régime. For times $\varepsilon \ll|t|<T_{*}$, the situation is different: the potential $U$ becomes negligible, while $V$ dictates the propagation. Like in the nonlinear setting, the transition between these two régimes is measured by the scattering operator associated to $U$.

The assumption $\sigma \geq \sigma_{0}(n)$ makes the nonlinear term short range. With our scaling for the nonlinearity, this perturbation is relevant only near the focus, where the potential is negligible, while the opposite occurs for $\varepsilon \ll|t| \leq T$.

The assumption on the potential $V$ in Theorem 4.3 is such that this result is complementary to the analysis in 9. It excludes the phenomenon of "total" refocusing. Indeed, the rays of geometric optics will all meet at one point for a time $t \neq 0$ if and only if $\delta_{j}=+1$ for all $j$ and the $\omega_{j}$ 's are pairwise rationally dependent. As discussed in 9], this is the only case where the nonlinear term could be relevant at leading order, past the initial neighborhood of size $\varepsilon$.

Remark. An explicit change of variable makes it possible to state the theorem when $\varphi\left(\frac{x}{\varepsilon}\right)$ is replaced by $\varphi\left(\frac{x-x_{0}}{\varepsilon}\right) e^{i x \cdot \xi_{0} / \varepsilon}$ (the phase factor $e^{i x \cdot \xi_{0} / \varepsilon}$ is actually not relevant, since the only assumption we make is $\varphi \in \Sigma)$, see [12].

Remark. We did not mention the case $\alpha=1>n \sigma$. Due to the lack of regularity of the nonlinearity $F(z)=|z|^{2 \sigma} z$, this régime can be analyzed more easily when the power nonlinearity is replaced by a Hartree-type nonlinearity. In [11, we study the asymptotics behavior as $\varepsilon \rightarrow 0$ of the solution $u^{\varepsilon}$ to

$$
i \varepsilon \partial_{t} u^{\varepsilon}+\frac{1}{2} \varepsilon^{2} \Delta u^{\varepsilon}=\frac{|x|^{2}}{2} u^{\varepsilon}+\varepsilon^{\alpha}\left(|x|^{-\gamma} *\left|u^{\varepsilon}\right|^{2}\right) u^{\varepsilon} \quad ; \quad u_{\mid t=0}^{\varepsilon}=f,
$$

with $\gamma>0, \alpha \geq 1$ and $x \in \mathbb{R}^{n}(n \geq 2)$. In the same spirit as above, the expected critical values for the parameters are $\alpha>1$ or $\alpha=1$ to measure the relevance 
of the nonlinearity outside of the focus, and $\alpha>\gamma$ or $\alpha=\gamma$ to decide whether the nonlinearity plays an important role at the caustic or not. When $\alpha=\gamma>1$, results similar to Theorem 4.2 are proved. When $\alpha=1>\gamma$, then as expected, the nonlinearity is relevant only away from the focus (and is measured by a slowly varying phase shift); the caustic crossing is described by the Maslov-Keller index. When $\alpha=\gamma=1$, both nonlinear effects mentioned above are expected, and the underlying scattering operator would be a long range scattering operator. This case is treated only through a formal computation.

Theorems 4.2 and 4.3 show that when the potential $V$ is of the form (3.1), the interactions between the linear dynamics and the nonlinear effects can be understood quite precisely. We mention two important limitations. First, we do not treat super-critical cases, such as $\lambda^{\varepsilon}=\varepsilon^{\alpha}$ with $\alpha<n \sigma$. The difficulty to treat such cases is not specific to the presence of the potential $V$, however. Second, a natural question is: what if $V$ is a general smooth sub-quadratic potential? Some answers are given in [12. As we already pointed out, the compatibility between linear and nonlinear analysis in 9, 12, relies essentially in Lemma 4.1] In [12, we ask: for which potentials $V$ can an operator of the form $\rho(t) e^{\phi / \varepsilon} \nabla_{x}\left(e^{\phi / \varepsilon} \cdot\right)$ (compare with Lemma 4.1 (ii)) commute with the linear part of (1.2) (point (i) in Lemma 4.1)? Easy computations show that this is possible only if $V$ is a second order polynomial (and when $\phi$ solves the eikonal equation (2.13). For initial data of the form (4.2), an interesting candidate as a substitute to $J^{\varepsilon}$ (which saves the day away from the focus in Theorems 4.2 and 4.3) would be the Heisenberg observable $U_{V}^{\varepsilon}(t) \frac{x}{\varepsilon} U_{V}^{\varepsilon}(-t)$.

Note that it coincides with $J^{\varepsilon}$ in the case $V(x)=\frac{|x|^{2}}{2}$ if $\frac{\pi}{2}$ is taken as a new time origin. Obviously, it satisfies the commutation property (i) in Lemma 4.1 It also yields a weighted Gagliardo-Nirenberg of the form stated in Lemma 4.1] (ii)', at least for $|t| \leq \delta$ small (the same as in Proposition [2.5). In Lemma 4.1 the only interest of point (ii) is to imply (ii)' and (ii)", so only the action of this Heisenberg observable on nonlinearities of the form $G\left(|z|^{2}\right) z$ remains to be understood. This issue seems to be connected to Egorov theorem; we have no answer to provide.

\section{Changing finite time BloW-UP}

Guided by the semi-classical analysis described above, we can understand the role of some potentials on the finite time blow-up phenomenon. In the linear semiclassical analysis, it is well known that the energy is carried by the bicharacteristic curves. On the other hand, blow-up for (2.1) is when the $L^{2}$-norm of $\nabla_{x} u$ becomes infinite, while the $L^{2}$-norm of $u$ is constant; heuristically, the energy of $u$ concentrates "somewhere". One can expect that if in the linear case, bicharacteristics meet, then the associated potential may encourage finite time blow-up in the nonlinear (focusing) case; the two effects cumulate. On the contrary, if the bicharacteristics spread out, then the potential may compete with the attractivity of the nonlinearity. We give several illustrations that provide a rigorous support for these ideas.

The first examples follow from the same heuristic, when one plays with the phase of the initial data instead of a potential in the equation. Consider the linear equation

$$
i \varepsilon \partial_{t} u^{\varepsilon}+\frac{1}{2} \varepsilon^{2} \Delta u^{\varepsilon}=0 \quad ; \quad u^{\varepsilon}(0, x)=f(x) e^{i b \frac{|x|^{2}}{2 \varepsilon}}, b \in \mathbb{R} .
$$

The Hamilton flow is given by

$$
\dot{t}=1 \quad ; \quad \dot{x}=\xi \quad ; \quad \dot{\xi}=\dot{\tau}=0 \quad ; \quad x_{\mid t=0}=x_{0} \quad ; \quad \xi_{\mid t=0}=b x_{0},
$$


and the classical trajectories are: $x(t)=x_{0}(1+b t)$. In particular $x\left(\frac{-1}{b}\right)=0$ for all $x_{0} \in \mathbb{R}$; if $b<0$, then rays meet at the origin in the future, while if $b>0$, they met at the origin in the past (see Figure 1). Following the above heuristic discussion,

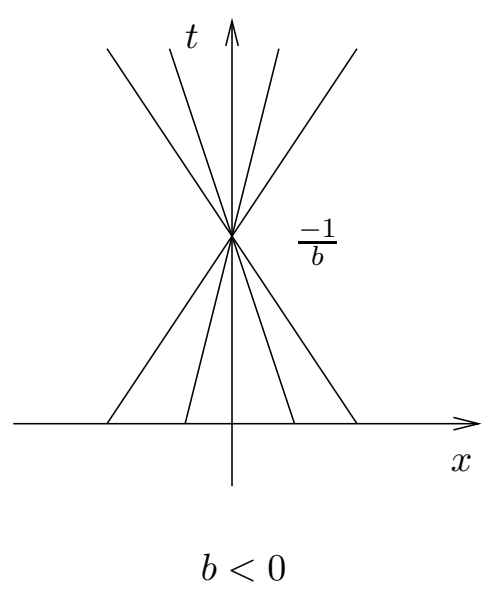

one may expect that quadratic initial oscillations alter the blow-up phenomenon in the nonlinear case. This is proved in 15: let $u$ solve (2.1) with $u_{0} \in \Sigma$, and suppose that blow-up may occur, that is $\lambda<0$ and $\sigma \geq \frac{2}{n}$. Denote $u^{b}$ the solution of (2.1) with initial datum $u_{0}^{b}(x)=u_{0}(x) e^{i b \frac{|x|^{2}}{2}}$. Then for $b \gg 1, u^{b}$ is global in the future $(t \geq 0)$. On the other hand, if $E_{0}\left(u_{0}\right)<0$, then we know from Proposition 2.3 that $u$ blows up at time $T>0$, say. It is proved in 15 that if $b<0$, then $u_{b}$ blows up at time $T^{b} \leq \frac{-1}{b}$. For $b<\frac{-1}{T}$, the blow up phenomenon occurs sooner for $u^{b}$ than for $u$. In the conformal case $\sigma=\frac{2}{n}$, the critical values for $b$ can be explicitly related to the blow-up time $T$ (see [15, 14]).

Consider now the equation

$$
i \varepsilon \partial_{t} u^{\varepsilon}+\frac{1}{2} \varepsilon^{2} \Delta u^{\varepsilon}=(E \cdot x) u^{\varepsilon} \quad ; \quad u^{\varepsilon}(0, x)=f(x) e^{i b \frac{|x|^{2}}{2 \varepsilon}}, b \in \mathbb{R} .
$$

The Hamilton flow is given by

$$
\dot{t}=1 \quad ; \quad \dot{x}=\xi \quad ; \quad \dot{\xi}=-E \quad ; \quad \dot{\tau}=0 \quad ; \quad x_{\mid t=0}=x_{0} \quad ; \quad \xi_{\mid t=0}=b x_{0},
$$

and the classical trajectories are: $x(t)=x_{0}(1+b t)-\frac{t^{2}}{2} E$. Rays meet at $\underline{x}=-\frac{1}{2 b^{2}} E$ at time $\frac{-1}{b}$. This is the same phenomenon as above, shifted in space. Recall that we saw in Section 2.3.1 that the introduction of a linear potential $E \cdot x$ does not change the time of blow-up, but only shifts the set where this occurs. Here again, intuition and results meet.

The last two cases we consider are isotropic harmonic potential and isotropic repulsive harmonic potential, with no initial rapid oscillation:

$$
i \varepsilon \partial_{t} u_{ \pm}^{\varepsilon}+\frac{1}{2} \varepsilon^{2} \Delta u_{ \pm}^{\varepsilon}= \pm \omega^{2} \frac{|x|^{2}}{2} u_{ \pm}^{\varepsilon} \quad ; \quad u_{ \pm}^{\varepsilon}(0, x)=f(x) .
$$

We have $x(t)=x_{0} h(t)$, where $h$ is given by (3.3). Thus, $x_{+}(t)=x_{0} \cos (\omega t)$, and $x_{-}(t)=x_{0} \cosh (\omega t)$. This is illustrated in Figure 2 Note the analogy with Figure 1] We already saw in Section 2.3.2 that the introduction of an isotropic 

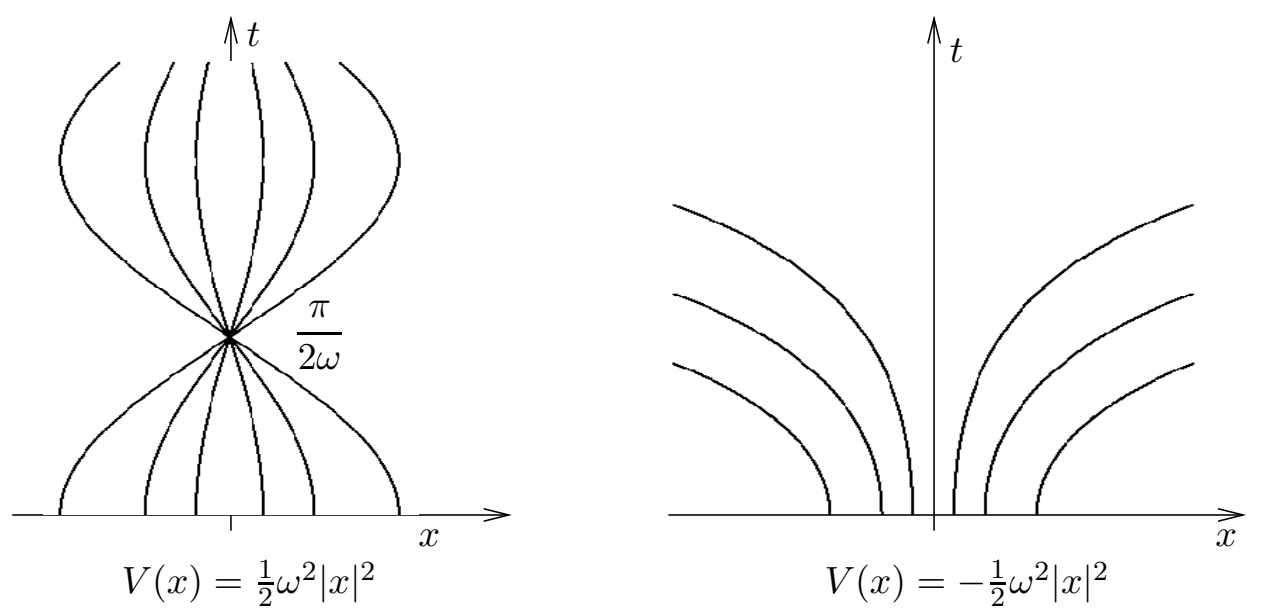

FIgURE 2. Geometry of rays: isotropic quadratic potential.

harmonic potential may anticipate the blow-up time when $\sigma=\frac{2}{n}$, just like quadratic oscillations in [15]. Similarly, the isotropic repulsive harmonic potential delays, or prevents, finite time blow-up.

To complete the picture, we have to study the case $\lambda\langle 0, \sigma\rangle \frac{2}{n}$.

Theorem 5.1 ([7, [8]). Let $u_{0} \in \Sigma, \lambda<0, \sigma \geq \frac{2}{n}$ and $\sigma<\frac{2}{n-2}$ if $n \geq 3$. Let $u_{ \pm}$ be the solutions of (1.2) with $\varepsilon=1$ and $V_{ \pm}(x)= \pm \omega^{2} \frac{|x|^{2}}{2}$.

1. If $E_{V_{+}}\left(u_{0}\right) \leq \frac{1}{2} \omega^{2}\left\|x u_{0}\right\|_{L^{2}}^{2}$, then $u_{+}$blows up at time $T_{+}^{\omega} \leq \frac{\pi}{2 \omega}$.

2. If the initial datum $u_{0}$ satisfies

$$
\frac{1}{2}\left\|\nabla u_{0}\right\|_{L^{2}}^{2}+\frac{\lambda}{\sigma+1}\left\|u_{0}\right\|_{L^{2 \sigma+2}}^{2 \sigma+2}<-\frac{\omega^{2}}{2}\left\|x u_{0}\right\|_{L^{2}}^{2},
$$

then $u_{-}$blows up in finite time, in the future or in the past.

3. If the initial datum $u_{0}$ satisfies

$$
\frac{1}{2}\left\|\nabla u_{0}\right\|_{L^{2}}^{2}+\frac{\lambda}{\sigma+1}\left\|u_{0}\right\|_{L^{2 \sigma+2}}^{2 \sigma+2}<-\frac{\omega^{2}}{2}\left\|x u_{0}\right\|_{L^{2}}^{2}-\omega\left|\operatorname{Im} \int \overline{u_{0}} x \cdot \nabla_{x} u_{0}\right|,
$$

then $u_{-}$blows up in finite time, in the future and in the past.

4. There exists $\omega_{1}>0$ such that for any $\omega \geq \omega_{1}$, the solution $u_{-}$is global in time.

Remark. Notice that in the first point, finite time blow-up occurs for a range of positive values of the energy (it was known that if $E_{V_{+}}<0$, then finite time blowup occurs, see [14]). This is in sharp contrast with the case of (2.1), where zero energy solutions may be global. When $\sigma=\frac{2}{n}$ the solitary wave $e^{i t} R(x)$, where $R$ is the ground state, solves (2.1), is global in time and has zero energy. Note that the condition in the first point also reads $E_{0}\left(u_{0}\right) \leq 0$ (but $E_{0}$ is not the energy associated to that equation!).

The proof of points 2 and 3 relies on the Zakharov-Glassey method, just like Proposition [2.3 so we shall not discuss it, and refer to [8]. The proofs of points 1 and 4 rely on two conservation laws, which are more precise than the conservation of energy, and can be viewed as analogs to the pseudo-conformal conservation law (2.6). These laws have a geometric meaning, since they "follow" the propagation. 
Lemma 5.2. Let $u_{0} \in \Sigma$, and $\sigma<\frac{2}{n-2}$ if $n \geq 3$. Let $u_{ \pm}$be the solutions of (1.2) with $\varepsilon=1$ and $V_{ \pm}(x)= \pm \omega^{2} \frac{|x|^{2}}{2}$. Introduce

$$
\begin{aligned}
& E_{+}^{1}(t):=\frac{1}{2}\left\|J_{+}(t) u_{+}\right\|_{L^{2}}^{2}+\frac{\lambda}{\sigma+1} \cos ^{2}(\omega t)\left\|u_{+}(t)\right\|_{L^{2 \sigma+2}}^{2 \sigma+2}, \\
& E_{+}^{2}(t):=\frac{\omega^{2}}{2}\left\|H_{+}(t) u_{+}\right\|_{L^{2}}^{2}+\frac{\lambda}{\sigma+1} \sin ^{2}(\omega t)\left\|u_{+}(t)\right\|_{L^{2 \sigma+2}}^{2 \sigma+2}, \\
& E_{-}^{1}(t):=\frac{1}{2}\left\|J_{-}(t) u_{-}\right\|_{L^{2}}^{2}+\frac{\lambda}{\sigma+1} \cosh ^{2}(\omega t)\left\|u_{-}(t)\right\|_{L^{2 \sigma+2}}^{2 \sigma+2}, \\
& E_{-}^{2}(t):=\frac{-\omega^{2}}{2}\left\|H_{-}(t) u_{-}\right\|_{L^{2}}^{2}-\frac{\lambda}{\sigma+1} \sinh ^{2}(\omega t)\left\|u_{-}(t)\right\|_{L^{2 \sigma+2}}^{2 \sigma+2},
\end{aligned}
$$

where $J_{+}$stands for $J$ when $V(x)=+\omega^{2} \frac{|x|^{2}}{2}$, and so on. Note that $E_{ \pm}^{1}(t)+E_{ \pm}^{2}(t) \equiv$ $E_{V_{ \pm}}$. We have:

$$
\begin{gathered}
\frac{d E_{+}^{1}}{d t}=\frac{\omega \lambda}{2 \sigma+2}(n \sigma-2) \sin (2 \omega t)\left\|u_{+}(t)\right\|_{L^{2 \sigma+2}}^{2 \sigma+2}, \\
\frac{d E_{-}^{1}}{d t}=\frac{\omega \lambda}{2 \sigma+2}(2-n \sigma) \sinh (2 \omega t)\left\|u_{-}(t)\right\|_{L^{2 \sigma+2}}^{2 \sigma+2} .
\end{gathered}
$$

Remark. These algebraic results can be proved in a classical way. Notice that one turn $V_{+}$into $V_{-}$by replacing $\omega$ with $i \omega$ (and vice versa).

The first point of Theorem 5 .1 follows easily. Assume that $E_{V_{+}}\left(u_{0}\right) \leq \frac{\omega^{2}}{2}\left\|x u_{0}\right\|_{L^{2}}^{2}$, and suppose that $u_{+}$exists in $\Sigma$ up to time $\frac{\pi}{2 \omega}$. Then $E_{+}^{1}(0)=E_{0}\left(u_{0}\right) \leq 0$, and from (5.11), if $\sigma \geq \frac{2}{n}$ and $\lambda<0$,

$$
\frac{d E_{+}^{1}}{d t} \leq 0, \quad \forall t \in\left[0, \frac{\pi}{2 \omega}\right] .
$$

This implies

$$
E_{+}^{1}\left(\frac{\pi}{2 \omega}\right) \leq 0
$$

But from the definition of $E_{+}^{1}$,

$$
E_{+}^{1}\left(\frac{\pi}{2 \omega}\right)=\frac{\omega^{2}}{2}\left\|x u_{+}\left(\frac{\pi}{2 \omega}\right)\right\|_{L^{2}}^{2},
$$

so this leads to a contradiction (unless $u_{+} \equiv 0$, which means that $u_{0} \equiv 0$ ). Therefore, $u_{+}$does not remains in $\Sigma$ up to time $\frac{\pi}{2 \omega}$. It is easy to conclude that this is so because there exists $T \leq \frac{\pi}{2 \omega}$ such that

$$
\lim _{t \rightarrow T}\left\|\nabla_{x} u_{+}(t)\right\|_{L^{2}}=\infty .
$$

Notice that the larger $\omega$, the sooner the blow-up. Note that the sufficient condition to have finite time blow-up does not depend on the value of $\omega>0$ though.

Even if the conditions 2 and 3 in Theorem [5.1 become void as $\omega \rightarrow+\infty$, this does not mean that the last point is true. In the conformal case $\sigma=\frac{2}{n}$, the last point is explicit, as we saw in Section 2.3.3 up to a characterization of finite time blow-up in this case where the potential is unbounded from below. Roughly speaking, the characterization of global existence is the same as in Theorem [2.1] The energy $E_{V_{-}}$is the sum of three terms, and the term corresponding to the nonlinearity is controlled by the gradient and the $L^{2}$ norm of the solution, which is conserved. Thus, if the gradient remains bounded in $L^{2}$, then each term of the energy remains bounded. Now the determinant of the matrix in (3.5) is constant, equal to -1 . Finally, to prove global existence, it is sufficient to check that the $L^{2}$ norm of $J_{-}(t) u$ remains bounded. 
We briefly sketch the proof of the last point of Theorem 5.1 and refer to 8 for details. The first step consists in noticing that the usual method to prove local existence still works for (1.2) with $\varepsilon=1$ and $V(x)=-\frac{\omega^{2}}{2}|x|^{2}$, and does not "see" the parameter $\omega \geq 0$. Indeed, as we already noticed, Mehler's formula yields the same dispersive estimate as in the case $\omega=0$, hence the same Strichartz estimates. Moreover, the operator $J_{-}$plays a role analog to that of $\nabla_{x}$ in the case of (2.1): it commutes with the linear part of the equation, acts on the nonlinearity like a derivative, and yields weighted Gagliardo-Nirenberg estimates with a weight uniformly bounded in $\omega \geq 0$ (see Lemma 4.1. (ii)', and recall that $\cosh x \geq 1$ ). Therefore, there exists $t_{0}>0$ independent of $\omega \geq 0$ such that $u_{-} \in C(]-2 t_{0}, 2 t_{0}[; \Sigma)$ and $A(t) u$ is bounded in $L^{\infty}\left(\left[-t_{0}, t_{0}\right]: L^{2}\right)$ uniformly in $\omega \geq 0$ for any $A \in\left\{I d, J_{-}, H_{-}\right\}$. Now integrate (5.2) between $t_{0}$ and $t>t_{0}$. Since $\lambda(2-n \sigma) \geq 0$,

$$
\begin{aligned}
E_{-}^{1}(t) & \leq E_{-}^{1}\left(t_{0}\right)+C \omega \int_{t_{0}}^{t} \sinh (2 \omega s)\left\|u_{-}(s)\right\|_{L^{2 \sigma+2}}^{2 \sigma+2} d s \\
& \leq C\left(C_{0}\right)+C \omega \int_{t_{0}}^{t} \frac{\sinh (2 \omega s)}{\cosh (\omega s)^{n \sigma}}\left\|J_{-}(s) u_{-}\right\|_{L^{2}}^{n \sigma} d s .
\end{aligned}
$$

The constants in the last estimate do not depend on $\omega$. Define

$$
y(t):=\sup _{t_{0} \leq s \leq t}\left\|J_{-}(s) u_{-}\right\|_{L^{2}}^{2}
$$

We have

$$
E_{-}^{1}(t) \leq C\left(C_{0}\right)+C y(t)^{n \sigma / 2} \int_{t_{0}}^{t} \frac{\sinh (2 \omega s)}{\cosh (\omega s)^{n \sigma}} d s \leq C\left(C_{0}\right)+\frac{C}{\cosh \left(\omega t_{0}\right)^{n \sigma-2}} y(t)^{n \sigma / 2} .
$$

We finally have

$$
y(t) \leq C\left(C_{0}\right)+\frac{C}{\cosh \left(\omega t_{0}\right)^{n \sigma-2}} y(t)^{n \sigma / 2} .
$$

For $\sigma>\frac{2}{n}$, we conclude by a bootstrap argument, since the constants and $t_{0}$ do not depend on $\omega>0$, and

$$
\cosh \left(\omega t_{0}\right) \underset{\omega \rightarrow+\infty}{\longrightarrow}+\infty .
$$

This yields a uniform bound for $\left\|J_{-}(t) u_{-}\right\|_{L^{2}}$, and proves global existence.

This proof relies on the evolution law (5.2), which seems to be bound to the case of isotropic potentials. A possible question is to ask whether a similar result holds when $V$ is of the form (3.1), with, say, $\delta_{1}=-1$ (recall that the $V$ is non-negative, things are rather well understood). An answer is given in [10]:

Theorem 5.3 ([10). Take $\varepsilon=1$. Let $n \geq 2, \lambda \in \mathbb{R}, \sigma \geq \frac{2}{n}$ with $\sigma<\frac{2}{n-2}$ if $n \geq 3$, and $u_{0} \in \Sigma$. Let $V$ be of the form (3.1) with $\delta_{1}=-1$, and denote

$$
\omega_{ \pm}=\max \left\{\omega_{j} ; \delta_{j}= \pm 1\right\} \quad\left(\omega_{+}=0 \text { if there is no } \delta_{j}=+1\right) .
$$

Then there exists $\Lambda=\Lambda\left(n, \sigma,|\lambda|,\left\|u_{0}\right\|_{\Sigma}\right)$ such that for

$$
\omega_{-} \geq \Lambda\left(1+\omega_{+}\right)+\frac{2 \sigma^{2}}{2-(n-2) \sigma}\left(1+\omega_{+}\right) \ln \left(1+\omega_{+}\right),
$$

the solution $u$ to (1.2) is global in time, $u \in C(\mathbb{R} ; \Sigma)$.

The statement can be summarized as follows: if the repulsive force is sufficiently strong compared to other effects (linear confinement is overcome if $\omega_{-} \gg \omega_{+}$, nonlinear effects are overcome if $\left.\omega_{-} \gg 1\right)$, then the solution is global. The strategy of the proof is as follows. First, in the same spirit as in [8, we analyze the local existence result, to bound from below the local existence time, in term of the parameters $\omega_{j}$. Then, we notice that we obtain a time at which $u$ is defined and small, and for which therefore the nonlinearity is not too strong. We consider the 
solution of the linear equation (1.2) with $\lambda=0$ ) that coincides with $u$ at that time. A continuity argument shows that $u$ cannot move away too much from this linear solution. Since the linear solution is global, so is $u$. Note that the nonlinear term in $\omega_{+}$in (5.3) is zero when $\omega_{+}=0$, that is when there is no confinement.

\section{More on GLObal EXistence}

In the last point of Theorem 5.1 and in Theorem 5.3 we saw that if we consider a quadratic potential which is sufficiently repulsive, then the solution to (1.2) is global. In that case, we even have scattering: the solution to (1.2) is asymptotically linear, as time becomes infinite.

It is proved in [8] that is the nonlinearity is defocusing $(\lambda>0)$, and the potential $V$ is the isotropic repulsive harmonic potential, then the solution $u$ of (1.2) is global, $u \in C(\mathbb{R} ; \Sigma)$. This follows from (5.2). We then have:

Proposition 6.1 ([8, 10]). Let $\varepsilon=1, \lambda, \sigma>0$, with $\sigma<\frac{2}{n-2}$ if $n \geq 3$.

1. Assume $V(x)=-\frac{\omega^{2}}{2}|x|^{2}$.

- For every $u_{-} \in \Sigma$, there exists a unique $u_{0} \in \Sigma$ such that the maximal solution $u \in C(\mathbb{R} ; \Sigma)$ to (1.2) satisfies

$$
\left\|U_{V}(-t) u(t)-u_{-}\right\|_{\Sigma} \underset{t \rightarrow-\infty}{\longrightarrow} 0 .
$$

- For every $u_{0} \in \Sigma$, there exists a unique $u_{+} \in \Sigma$ such that the maximal solution $u \in C(\mathbb{R} ; \Sigma)$ to (1.2) satisfies

$$
\left\|U_{V}(-t) u(t)-u_{+}\right\|_{\Sigma} \underset{t \rightarrow+\infty}{\longrightarrow} 0 .
$$

2. Suppose that $V$ is of the form (3.1) with $\delta_{1}=-1$.

- For every $u_{-} \in \Sigma$, there exist $T$ finite and a unique $\left.\left.u \in C(]-\infty, T\right] ; \Sigma\right) \cap$ $\left.\left.L^{q}(]-\infty, T\right] ; L^{2 \sigma+2}\left(\mathbb{R}^{n}\right)\right)$, where $q=\frac{4 \sigma+4}{n \sigma}$, solution to (1.2) such that

$$
\left\|U_{V}(-t) u(t)-u_{-}\right\|_{\Sigma} \underset{t \rightarrow-\infty}{\longrightarrow} 0 \text {. }
$$

- Let $u_{0} \in \Sigma$. Then taking $\Lambda$ larger in (5.3) if necessary, there exists a unique $u_{+} \in \Sigma$ such that the maximal solution $u \in C(\mathbb{R} ; \Sigma)$ to (1.2) satisfies

$$
\left\|U_{V}(-t) u(t)-u_{+}\right\|_{\Sigma} \underset{t \rightarrow+\infty}{\longrightarrow} 0 .
$$

Notice that unlike in Proposition 2.4 there is no additional assumption on $\sigma$, simply $\sigma>0$ : all power-like nonlinearities are short range for the Hamiltonian $H_{V}$ under our hypotheses.

The key ingredient of the proof consists in noticing that in the two cases, global existence follows from the boundedness of $J(t) u$ in $L^{\infty}\left(\mathbb{R} ; L^{2}\right)$. The weighted Gagliardo-Nirenberg inequality of Lemma 4.1 yields exponential decay for $u$, and any positive power of an exponentially decreasing functions is integrable at infinity.

The restriction $u \in C(]-\infty, T] ; \Sigma)$ in the first part of point 2 comes from the fact that in general, we cannot prove that even if $\lambda>0$, the solution $u$ is defined globally in time.

\section{REFERENCES}

1. J. E. Avron and I. W. Herbst, Spectral and scattering theory of Schrödinger operators related to the Stark effect, Comm. Math. Phys. 52 (1977), no. 3, 239-254.

2. C. C. Bradley, C. A. Sackett, J. J. Tollett, and R. G. Hulet, Evidence of Bose-Einstein condensation in an atomic gas with attractive interactions, Phys. Rev. Lett. 75 (1995), 16871690.

3. J. C. Bronski and R. L. Jerrard, Soliton dynamics in a potential, Math. Res. Lett. 7 (2000), no. $2-3,329-342$. 
4. N. Burq, P. Gérard, and N. Tzvetkov, Strichartz inequalities and the nonlinear Schrödinger equation on compact manifolds, Amer. J. Math., to appear.

5. - An instability property of the nonlinear Schrödinger equation on $S^{d}$, Math. Res. Lett. 9 (2002), no. 2-3, 323-335.

6. R. Carles, Critical nonlinear Schrödinger equations with and without harmonic potential, Math. Models Methods Appl. Sci. 12 (2002), no. 10, 1513-1523.

7. _ Remarks on nonlinear Schrödinger equations with harmonic potential, Ann. Henri Poincaré 3 (2002), no. 4, 757-772.

8. __ Nonlinear Schrödinger equations with repulsive harmonic potential and applications, SIAM J. Math. Anal. 35 (2003), no. 4, 823-843.

9. _ Semi-classical Schrödinger equations with harmonic potential and nonlinear perturbation, Ann. Inst. H. Poincaré Anal. Non Linéaire 20 (2003), no. 3, 501-542.

10. Global existence results for nonlinear Schrödinger equations with quadratic potentials, preprint, arxiv:math.AP/0405197, 2004.

11. R. Carles, N. J. Mauser, and H. P. Stimming, (Semi)classical limit of the Hartree equation with harmonic potential, preprint, arXiv:math.AP/0405370, 2004.

12. R. Carles and L. Miller, Semiclassical Nonlinear Schrödinger equations with potential and focusing initial data, Osaka J. Math. 41 (2004), no. 3, to appear.

13. R. Carles and Y. Nakamura, Nonlinear Schrödinger equations with Stark potential, Hokkaido Math. J., to appear.

14. T. Cazenave, Semilinear Schrödinger equations, Courant Lecture Notes in Mathematics, vol. 10, New York University Courant Institute of Mathematical Sciences, New York, 2003.

15. T. Cazenave and F. Weissler, Rapidly decaying solutions of the nonlinear Schrödinger equation, Comm. Math. Phys. 147 (1992), 75-100.

16. W. Craig, T. Kappeler, and W. Strauss, Microlocal dispersive smoothing for the Schrödinger equation, Comm. Pure Appl. Math. 48 (1995), no. 8, 769-860.

17. B. Deconinck, B. Frigyik, and J. N. Kutz, Dynamics and stability of Bose-Einstein condensates: the nonlinear Schrödinger equation with periodic potential, J. Nonlinear Sci. 12 (2002), no. 3, 169-205.

18. S.-I. Doi, Smoothing effects for Schrödinger evolution equation and global behavior of geodesic flow, Math. Ann. 318 (2000), 355-389.

19. J. J. Duistermaat, Oscillatory integrals, Lagrange immersions and unfolding of singularities, Comm. Pure Appl. Math. 27 (1974), 207-281.

20. N. Dunford and J. T. Schwartz, Linear operators. Part II: Spectral theory. Self adjoint operators in Hilbert space, With the assistance of William G. Bade and Robert G. Bartle, Interscience Publishers John Wiley \& Sons New York-London, 1963.

21. R. P. Feynman and A. R. Hibbs, Quantum mechanics and path integrals (International Series in Pure and Applied Physics), Maidenhead, Berksh.: McGraw-Hill Publishing Company, Ltd., 365 p., 1965.

22. D. Fujiwara, A construction of the fundamental solution for the Schrödinger equation, J. Analyse Math. 35 (1979), 41-96.

23. Remarks on the convergence of the Feynman path integrals, Duke Math. J. 47 (1980), no. $3,559-600$.

24. J. Ginibre and G. Velo, On a class of nonlinear Schrödinger equations. I The Cauchy problem, general case, J. Funct. Anal. 32 (1979), 1-32.

25. _ On a class of nonlinear Schrödinger equations. II Scattering theory, general case, J. Funct. Anal. 32 (1979), 33-71.

26. - The global Cauchy problem for the nonlinear Schrödinger equation revisited, Ann. Inst. H. Poincaré Anal. Non Linéaire 2 (1985), 309-327.

27. R. T. Glassey, On the blowing up of solutions to the Cauchy problem for nonlinear Schrödinger equations, J. Math. Phys. 18 (1977), 1794-1797.

28. N. Hayashi and Y. Tsutsumi, Remarks on the scattering problem for nonlinear Schrödinger equations, Differential equations and mathematical physics (Birmingham, Ala., 1986), Lectures Notes in Math., vol. 1285, Springer, Berlin, 1987, pp. 162-168.

29. L. Hörmander, Symplectic classification of quadratic forms, and general Mehler formulas, Math. Z. 219 (1995), no. 3, 413-449.

30. A. Jensen and T. Kato, Spectral properties of Schrödinger operators and time decay of the wave functions, Duke Math. J. 46 (1979), no. 3, 583-611.

31. $\mathrm{R}$ Johnson and $\mathrm{X}$. Pan, On an elliptic equation related to the blow-up phenomenon in the nonlinear Schrödinger equation, Proc. Roy. Soc. Edinburgh Sect. A 123 (1993), no. 4, 763782.

32. L. Kapitanski and I. Rodnianski, Regulated smoothing for Schrödinger evolution, Internat. Math. Res. Notices (1996), no. 2, 41-54. 
33. O. Kavian and F. Weissler, Self-similar solutions of the pseudo-conformally invariant nonlinear Schrödinger equation, Michigan Math. J. 41 (1994), no. 1, 151-173.

34. M. Keel and T. Tao, Endpoint Strichartz estimates, Amer. J. Math. 120 (1998), no. 5, 955980.

35. S. Keraani, Semiclassical limit for a class ofNonlinear Schrödinger Equations with potential, Comm. Part. Diff. Eq. 27 (2002), no. 3-4, 693-704.

36. S. Klainerman, Uniform decay estimates and the Lorentz invariance of the classical wave equation, Comm. Pure Appl. Math. 38 (1985), no. 3, 321-332.

37. E. B. Kolomeisky, T. J. Newman, J. P. Straley, and X. Qi, Low-dimensional Bose liquids: Beyond the Gross-Pitaevskii approximation, Phys. Rev. Lett. 85 (2000), no. 6, 1146-1149.

38. F. Merle, Construction of solutions with exactly $k$ blow-up points for the Schrödinger equation with critical nonlinearity, Comm. Math. Phys. 129 (1990), no. 2, 223-240.

39. F. Merle and P. Raphaël, Sharp upper bound on the blow-up rate for the critical nonlinear Schrödinger equation, Geom. Funct. Anal. 13 (2003), no. 3, 591-642.

40. K. Nakanishi and T. Ozawa, Remarks on scattering for nonlinear Schrödinger equations, NoDEA Nonlinear Differential Equations Appl. 9 (2002), no. 1, 45-68.

41. U. Niederer, The maximal kinematical invariance groups of Schrödinger equations with arbitrary potentials, Helv. Phys. Acta 47 (1974), 167-172.

42. F. Nier, A semi-classical picture of quantum scattering, Ann. Sci. École Norm. Sup. (4) 29 (1996), no. 2, 149-183.

43. F. Nier and A. Soffer, Dispersion and Strichartz estimates for some finite rank perturbations of the Laplace operator, J. Funct. Anal. 198 (2003), no. 2, 511-535.

44. Y.-G. Oh, Cauchy problem and Ehrenfest's law of nonlinear Schrödinger equations with potentials, J. Diff. Eq. 81 (1989), no. 2, 255-274.

45. L. Pitaevskii and S. Stringari, Bose-Einstein condensation, International Series of Monographs on Physics, vol. 116, The Clarendon Press Oxford University Press, Oxford, 2003.

46. M. Reed and B. Simon, Methods of modern mathematical physics. II. Fourier analysis, selfadjointness, Academic Press [Harcourt Brace Jovanovich Publishers], New York, 1975.

47. D. Robert, Autour de l'approximation semi-classique, Progress in Mathematics, vol. 68, Birkhäuser Boston Inc., Boston, MA, 1987.

48. I. Rodnianski and W. Schlag, Time decay for solutions of Schrödinger equations with rough and time-dependent potentials, Invent. Math. 155 (2004), no. 3, 451-513.

49. A. V. Rybin, G. G. Varzugin, M. Lindberg, J. Timonen, and R. K. Bullough, Similarity solutions and collapse in the attractive Gross-Pitaevskii equation, Phys. Rev. E (3) 62 (2000), no. 5, part A, 6224-6228.

50. G. Staffilani and D. Tataru, Strichartz estimates for a Schrödinger operator with nonsmooth coefficients, Comm. Partial Differential Equations 27 (2002), no. 7-8, 1337-1372.

51. R. Strichartz, Restrictions of Fourier transforms to quadratic surfaces and decay of solutions of wave equations, Duke Math. J. 44 (1977), no. 3, 705-714.

52. W. Thirring, A course in mathematical physics. Vol. 3, Springer-Verlag, New York, 1981, Quantum mechanics of atoms and molecules, Translated from the German by Evans M. Harrell, Lecture Notes in Physics, 141.

53. Y. Tsutsumi, $L^{2}$-solutions for nonlinear Schrödinger equations and nonlinear groups, Funkcial. Ekvac. 30 (1987), no. 1, 115-125.

54. M. I. Weinstein, Nonlinear Schrödinger equations and sharp interpolation estimates, Comm. Math. Phys. 87 (1982/83), no. 4, 567-576.

55. K. Yajima, Existence of solutions for Schrödinger evolution equations, Comm. Math. Phys. 110 (1987), 415-426.

56. L Smoothness and non-smoothness of the fundamental solution of time dependent Schrödinger equations, Comm. Math. Phys. 181 (1996), no. 3, 605-629.

57. K. Yajima and G. Zhang, Smoothing property for Schrödinger equations with potential superquadratic at infinity, Comm. Math. Phys. 221 (2001), no. 3, 573-590.

E-mail address: remi.carles@math.univ-rennes1.fr 